\title{
Two-dimensional spectroscopy of a sunspot
}

\section{Properties of the penumbral fine structure}

\author{
A. Tritschler, R. Schlichenmaier, L. R. Bellot Rubio, and the KAOS Team
}

Kiepenheuer-Institut für Sonnenphysik, Schöneckstr. 6, 79104 Freiburg, Germany

Received 5 August 2003 / Accepted 7 November 2003

\begin{abstract}
We investigate the properties of the fine structure of a sunspot penumbra based on spectroscopic measurements with high spectral $(\lambda / \delta \lambda=250000)$ and high spatial $(\approx 0.5 \operatorname{arcsec})$ resolution. The magnetically insensitive Fe I $557.6 \mathrm{~nm}$ line is used to probe the penumbral atmosphere. The data was taken at the German Vacuum Tower Telescope with the 2D-spectrometer TESOS, taking advantage of the recently installed Kiepenheuer Adaptive Optics System (KAOS). The field of view covers a sunspot located at $23^{\circ}$ off the disk center and its immediate surroundings. The penumbral structure is studied by means of maps computed for the line-of-sight velocity, the line width, the equivalent width and the line depression. Line-of-sight velocities are derived from the Doppler shifts at different bisector levels. From these maps we infer the flow field geometry and study the azimuthal and radial dependences of the line parameters. Our findings can be summarized as follows: (a) the flow pattern has a conspicuous filamentary structure in the deep photospheric layers and is rather diffuse in the high layers. (b) The flow field slightly spreads and fans out with height. (c) The flow geometry confirms the presence of an upflow component in the inner penumbra and a downflow component in the middle and outer penumbra. (d) We find an enhanced brightness of the mid-penumbra ("bright ring") in the line wings, but not in the continuum or line core. (e) The azimuthal average of the equivalent width, the line width and the absolute flow velocity increase with radial distance within the penumbra. (f) Smallscale variations of the equivalent width and the line width on the center-side penumbra are co-spatial and correlated with (blue-shifted) fluctuations in the line-of-sight velocity. (g) Inner limb-side penumbral grains are associated with blue-shifts of $v \leq-400 \mathrm{~m} \mathrm{~s}^{-1}$, indicating upflows. (h) One umbral dot in our sample is associated with a blue-shift of $v=-200 \mathrm{~m} \mathrm{~s}^{-1}$.
\end{abstract}

Key words. Sun: activity - photosphere - sunspots - spectroscopy

\section{Introduction}

Recent observations of sunspots with unprecedented spatial resolution (Scharmer et al. 2002) disclose a rather unexpected picture of the sunspot fine structure and show quite plainly that high spatial resolution is a very important requirement for solar observations. In addition to two-dimensional imaging at the diffraction limit, spectroscopy at the highest spatial resolution is needed in order to understand the physical cause of this fine structure.

With regard to these demands, we selected a magnetically insensitive line and used the combination of the upgraded 2D-spectrometer TESOS (Kentischer et al. 1998; Tritschler et al. 2002) and the Kiepenheuer Adaptive Optics System KAOS (Berkefeld et al. 2003; von der Lühe et al. 2003) to accomplish sunspot observations, that for the first time feature high spectral and high spatial resolution at the same time.

The velocity field of a sunspot in the photosphere is dominated by the conspicuous penumbral Evershed flow, first

Send offprint requests to: A. Tritschler,

e-mail: ali@kis.uni-freiburg.de

* The KAOS team includes: D. Soltau, T. Berkefeld, T. Schelenz. observed by Evershed (1909) and interpreted as a horizontal and radially directed outflow. In photospheric lines, the effect shows up as a line shift (St. John 1913) and is accompanied by a line asymmetry: line wings on either side of the line are stronger shifted in wavelength than the line core. The Evershed effect is height-dependent (Boerner \& Kneer 1992; Rouppe van der Voort 2002). In the photosphere, both the line-core shift and the asymmetry depend on the formation height in such a way that the line shift decreases while the line asymmetry increases with increasing formation height (e.g. Stellmacher \& Wiehr 1980; Balthasar et al. 1997). Spectroscopic observations probing near-continuum layers indicate that the line-of-sight component of the velocity increases with radial distance and reaches a maximum near the outer boundary of the penumbra, and then ceases more or less abruptly (Wiehr et al. 1986; Title et al. 1993; Hirzberger \& Kneer 2001). By contrast, spectropolarimetric observations in the infrared suggest that the flow continues well beyond the outer penumbral boundary (e.g. Solanki et al. 1994; Bellot Rubio et al. 2002; Bellot Rubio 2003; Mathew et al. 2003). Observations in weak lines give evidence for vertical flow components in the form of upflow and downflow patches 
located in the inner penumbra and the outer penumbra or beyond, respectively (Stanchfield et al. 1997; Westendorp Plaza et al. 1997; Schlichenmaier \& Schmidt 1999; del Toro Iniesta et al. 2001). The verification of a vertical flow component is crucial for the question of mass conservation of the Evershed flow.

The discrepancies found in the correlation of the horizontal outflow with continuum intensity, magnetic field strength and magnetic field inclination are not fully clarified. Most findings agree upon that the strongest flows are predominantly confined to the dark filaments (Wiehr \& Degenhardt 1992; Title et al. 1993; Rimmele 1995a; Stanchfield et al. 1997; Westendorp Plaza et al. 2001), where the magnetic field appears to be more horizontal (Degenhardt \& Wiehr 1991; Schmidt et al. 1992; Westendorp Plaza et al. 2001).

The relation between field strength and inclination is less conclusive. Further, there is only little knowledge about how the velocity field is linked to the behavior of the line width and the equivalent width, a subject that has been already mentioned by Johannesson (1993) and Rimmele (1995a,b) but not studied in detail. Also the thermal structure of sunspot penumbrae is poorly known and still a subject of debate (del Toro Iniesta et al. 1994; Westendorp Plaza et al. 2001; Balasubramaniam 2002; Rouppe van der Voort 2002).

For more details and references, the reader is referred to the reviews given by Martinez Pillet (1997), Schlichenmaier (2003) and Bellot Rubio (2003). An extensive overview of sunspot related issues can be found in Solanki (2003).

On the basis of our high-resolution observations we aim to contribute to the above-mentioned debates with a series of publications. In this investigation we concentrate on the presentation of the data set and the subsequent analysis in terms of maps of line-of-sight (LOS) velocity, continuum intensity, line width and equivalent width, with special emphasis on the penumbral fine structure. The line parameters are studied with regard to their morphology, their dependence with radial distance from the center of the umbra (Sect. 5.2), and their variation with azimuth at a constant radial distance (Sect. 5.3). In Sect. 5.4 we infer the flow geometry within the penumbra on the basis of the azimuthal variation of the LOS component of the velocity, giving further evidence for the presence of a downflow component within the penumbra. The differences between the limbside and center-side penumbra are illustrated and discussed in Sect. 5.5. Section 6 summarizes our findings.

In the second paper of this series (Schlichenmaier et al. 2004, this issue) we elaborate on a bisector analysis and in a forthcoming publications we perform an inversion of the observed line profiles to study the thermal and kinematic structure of the penumbra.

\section{Observations}

Observations have been carried out at the Vacuum Tower Telescope (VTT) at the Observatorio del Teide on Tenerife, between July 2 and July 6, 2002. The observations were performed using the upgrade configuration of the two-dimensional Fabry-Perot spectrometer TESOS (Kentischer et al. 1998; von der Lühe \& Kentischer 2000; Tritschler et al. 2002).
TESOS was used in its high resolution mode with a total field-of-view of $42 \times 42 \mathrm{arcsec}^{2}$, an image scale of $0.089 \operatorname{arcsec}$ pixel $^{-1}$ and a spectral resolution of 250000 . The data set consists of line scans obtained in the neutral iron line at $557.61 \mathrm{~nm}$. Each scan consists of 100 exposures, taken at equidistant wavelength points of $0.84 \mathrm{pm}$ distance step width, corresponding to overcritical spectral sampling. The narrowband pre-filter had a full width at half maximum of $1.1 \mathrm{~nm}$ and a transmission of $70 \%$. The exposure time and the cycle time was $10 \mathrm{~ms}$ and $37 \mathrm{~s}$, respectively. The broad-band channel of TESOS was equipped with a $G$-band filter centered at $431.1 \mathrm{~nm}$ with a width of $0.39 \mathrm{~nm}$. The broad-band data are employed only to correct for image shifts and to ensure that transparency fluctuations have not taken place during the scan.

Real-time seeing correction and image stabilization was accomplished by the Kiepenheuer Adaptive Optics System (KAOS) installed at the VTT since beginning of 2002. During our period of observations KAOS came into operation in combination with a scientific observing campaign for the first time. For a detailed description of the system and its setup the reader is referred to Soltau et al. (2002) and von der Lühe et al. (2003).

On 5 consecutive days mainly pores but also sunspots have been observed. In the present work we concentrate on the sunspot observations obtained on the 5th of July. The selected f-spot is part of a decaying sunspot group (AR 0019) located at $\theta=22.6^{\circ}$ corresponding to $\mu=0.92$. Figure 1 (top) shows a typical narrow-band filtergram taken in the continuum of the $557.61 \mathrm{~nm}$ line. The image is slightly unsharped masked. The surroundings outside the field-of-view of TESOS are displayed in the magnetogram and the continuum image taken by MDI (bottom). In particular, the MDI continuum data shows that the filament-like intrusion into the umbra evolved from a lightbridge and that the small spot next to the main target separated on the day before our observation.

Two complementary scans of the granulation next to the observed spot (with the spot still inside the field-of-view of TESOS) and in the direction perpendicular to the center-tolimb axis were taken $7 \mathrm{~min}$ after the sunspot data. These scans are mainly used for the velocity calibration (see Sect. 4.1).

The Fe I $557.61 \mathrm{~nm}$ is a fairly strong, magnetically insensitive line $(g=0)$, and is commonly used for velocity measurements. This line is often quoted to be insensitive to temperature. An analysis based on response functions indicates that this statement is not true. Fe I $557.61 \mathrm{~nm}$ may not be as sensitive as other lines, but it still reacts to temperature changes. In a forthcoming publication, this fact is exploited to infer the thermal structure of the spot at different heights in the atmosphere.

In Fig. 2, spatially averaged umbral (dashed), penumbral (dotted) and quiet photosphere (solid) line profiles are displayed. The profiles are given in observed units (left) and normalized to the local continuum intensity (right). Due to the presence of a band of $\mathrm{TiO}$ lines, the iron line is blended by one of these lines in the blue wing at $\sim 557.59 \mathrm{~nm}$. The blend mainly influences the umbral signal but is negligible under conditions that are typical for the penumbra and the quiet photosphere (see Fig. 2).

In order to visualize the quality of our data set and for later considerations, Fig. 3 shows a sequence of individual 

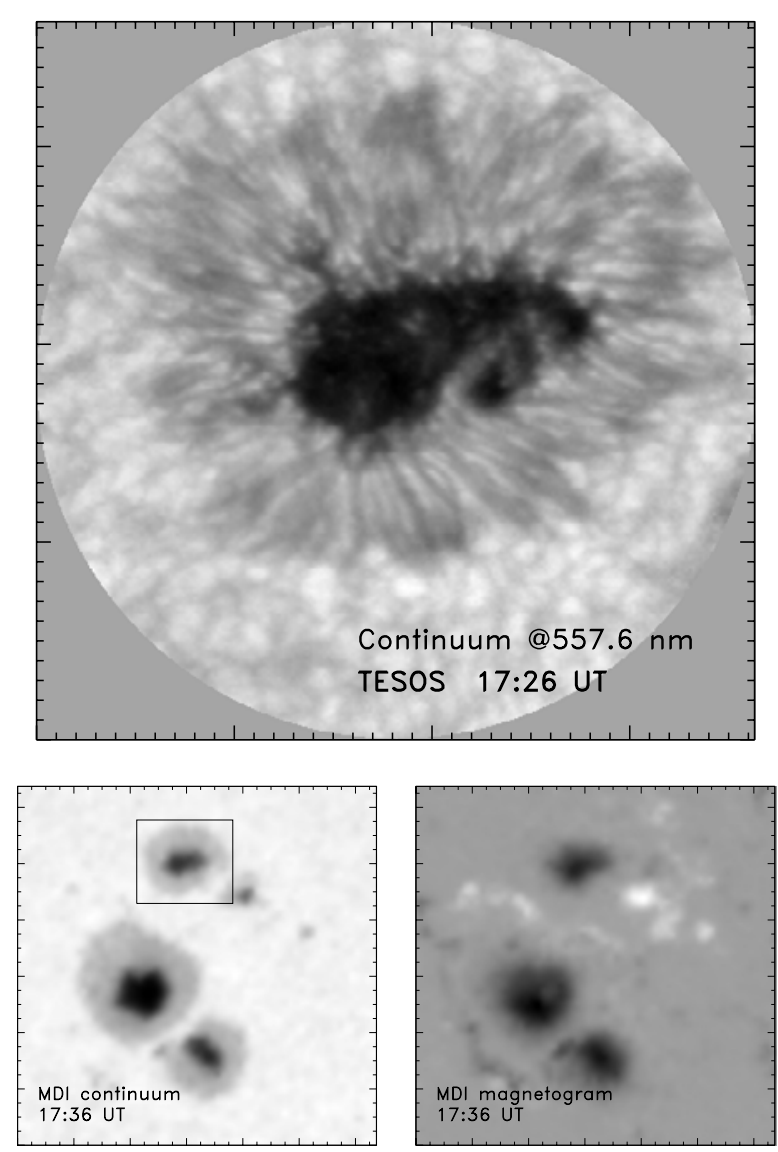

Fig. 1. Top: narrow-band continuum image (near $557.61 \mathrm{~nm}$ ) of the spot observed on July 5, 2002. One minor tick mark corresponds to 2 arcsec. Bottom: MDI continuum image and magnetogram of AR 0019 on the 5th July. The black rectangular in the MDI continuum image highlights the observed sunspot. The MDI continuum image and the magnetogram are adapted to the orientation of TESOS. One minor tick mark corresponds to 5 arcsec.

filtergrams covering every second wavelength step from position \#28 to \#72 out of 100 wavelength points. Filtergram \#50 corresponds approximately to the line core. The wavelength positions are marked in the line profile of the mean granular intensity shown in the lower right plot. The upper left image shows the best continuum filtergram (\#13) in the data set.

\section{Data reduction}

Standard calibration procedures like dark subtraction and gain table correction are applied. Grid targets in the main focus at the entrance of TESOS are used to determine the actual rotation and image scale differences between the filtergrams and the simultaneously taken broad-band images. The correction for these differences are done with respect to the filtergrams. Pointing errors during the scan are derived from a correlation analysis of the broad-band images ( $G$-Band) and are compensated for by a rigid alignment of the filtergrams with respect to the broad-band images. Due to the AO, the effects of image distortion are minimized. Therefore and in order to avoid the loss of information induced by interpolation processes, no
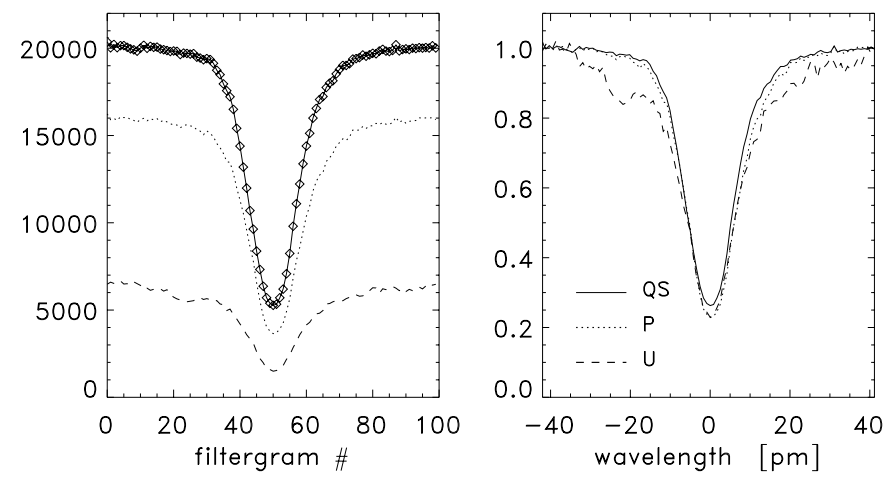

Fig. 2. Left: spatially averaged umbral (dashed), penumbral (dotted) and quiet photosphere (solid) line profiles (arbitrary units). The $\diamond$-symbols in the quiet photosphere profile indicate the the 100 wavelength points. Right: line profiles given in units of the mean local continuum intensity.

destretch is done. For the same reason, image drifts during a scan are corrected only with pixel accuracy.

\subsection{Modulation pattern}

The measured line profiles show an intensity modulation (cf. Tritschler et al. 2002). The period and amplitude of the intensity variation is constant in space and time. The effect is wavelength dependent and its amplitude amounts to $\sim 5 \%$ of the mean local continuum intensity in our measurements at $557.6 \mathrm{~nm}$. In order to remove the modulation we proceeded as follows. We perform an inversion to the observed mean line profile of the quiet photosphere by using the SIR (Stokes Inversion based on Response functions) code developed by Ruiz Cobo \& del Toro Iniesta (1992). The two-component model of the quiet-sun of Borrero \& Bellot Rubio (2002) is used in the inversion to account for the line asymmetry $(C$ shape) of the mean quiet-sun line profile. The result is a nonlinear least-squares fit to the observed profile. From the direct comparison of the fitted and the observed profile the modulation pattern is extracted. The derived modulation provides correction factors for each wavelength position and is applied at each spatial position in a subsequent step. Due to possible imperfections of the correction, residual seeing influences and readout noise, the rms-variation in the continuum intensity of quiet-sun line profiles after the correction amounts to $\sim 0.5-1 \%$.

\subsection{Parasitic light}

Parasitic light stems mainly from the contribution of true scatter processes inside the instrument and from false light that falls into the instrument or originates from reflections at optical elements in the light beam. Further, back-reflections of the light between the etalons and/or the interference pre-filter (ghosts) give rise to side lobes within the passband and produce an additional contribution. To estimate the influence of these effects on our measurements we perform scattered light and ghost measurements with a central obscuration in the focal plane at the entrance of TESOS as described in Tritschler et al. (2002). The combined effect of both ghosts and scattered light inside 


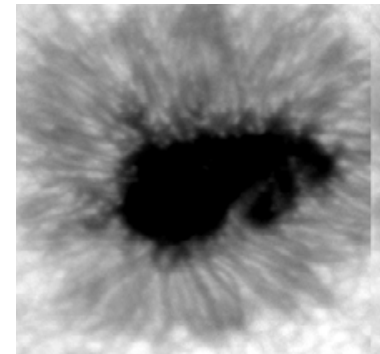

13

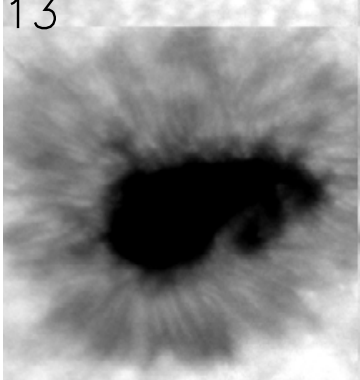

36

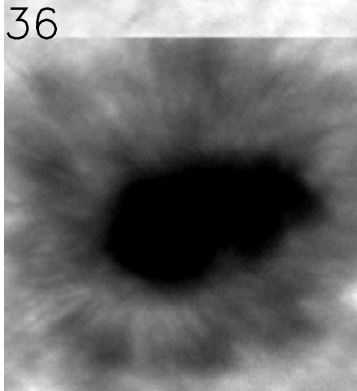

46

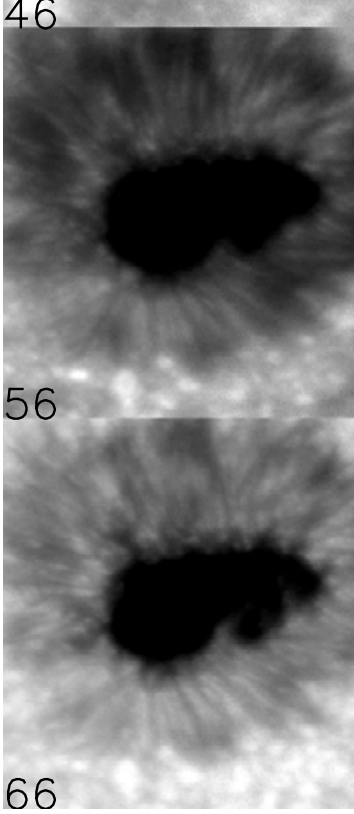

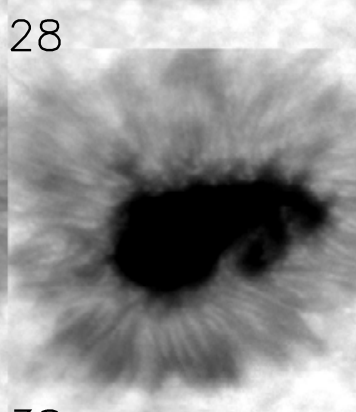

38

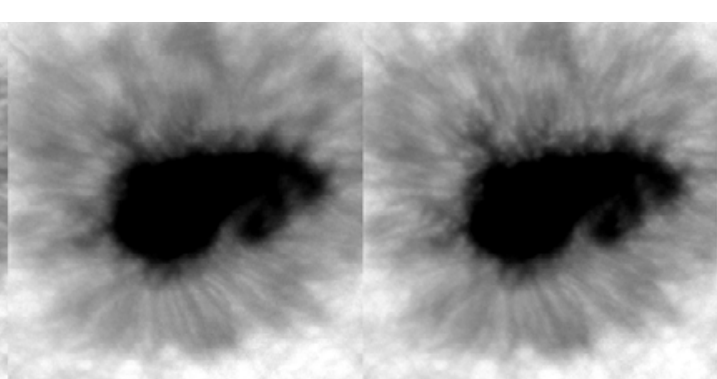

30

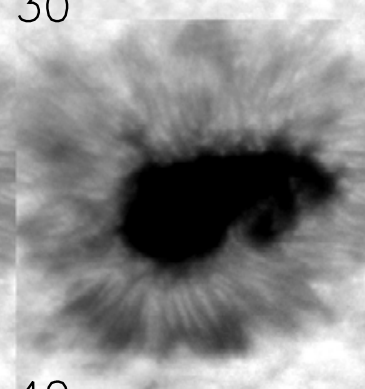

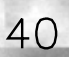
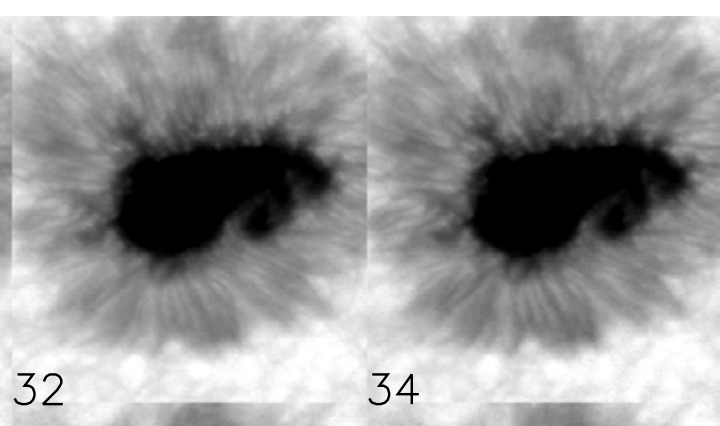

32

34
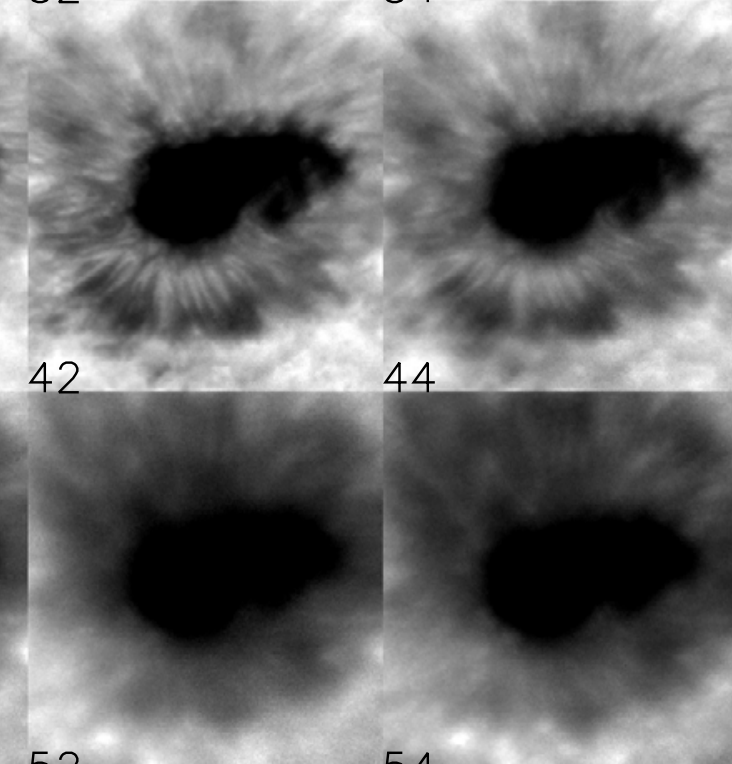

50

52

54

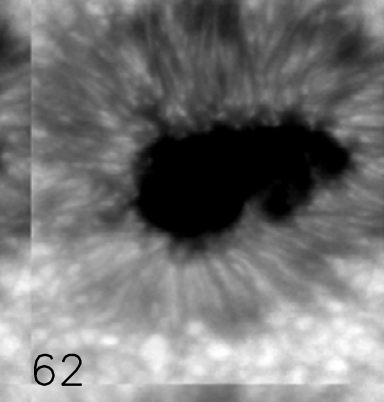

64

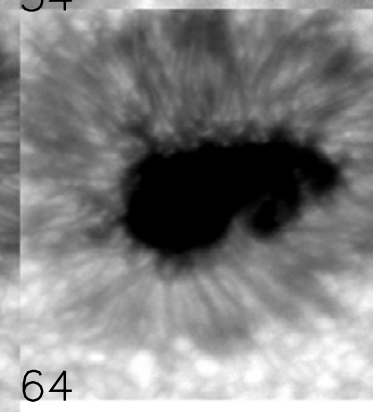

60
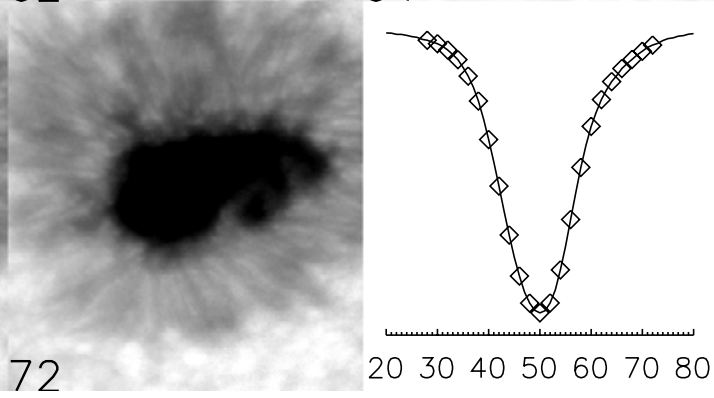

Fig. 3. Scan through the Fe I $557.6 \mathrm{~nm}$ line. The upper left image displays the best continuum filtergram in the wavelength scan (\#13). The subsequent filtergrams show every second wavelength step from position \#28 to \#72 as indicated in the lower right plot.

TESOS is estimated from the mean residual intensity found on the obscuration. For the continuum and the line core this amounts to $\sim 6.0 \%$ and $\sim 2.5 \%$ of the mean continuum intensity outside the obscuration. On average, $\sim 5.5 \%$ of the continuum intensity contributes to each filtergram in form of ghosts and scattered light. In the narrow-band channel the observed umbral continuum brightness is in the range $0.28-0.38$ in units of the mean intensity outside the spot. Since our stray light and ghost measurements only refer to TESOS itself, the measured umbral brightness suggests an additional stray light influence of at least $10 \%$ that originates from the telescope and the sky. However, umbral brightness is believed to depend also on temperature and the number density of umbral dots, conditions that might differ from spot to spot. 
With regard to our investigation it is important to note that even a $15 \%$ contamination of the line profiles due to stray light does not significantly change the shape of the penumbral bisectors nor the relative behavior of quantities like e.g. the line width or equivalent width. This was checked by means of the following experiment. We took penumbral profiles, added different percentages of straylight to them and calculated the bisectors before and after the addition. As an estimation of the stray light profile we took a spatially averaged line profile of the spot surroundings as well as of the penumbra. The stray light of course changes the bisector velocities, but the shape of the bisector itself is not changed significantly as long as the percentage of stray light is not larger than $15 \%$.

\subsection{Observing conditions}

Seeing conditions during the observing period have been variable but the AO-system assured the correction for image motion and image degradation due to seeing effects. In order to estimate the importance of residual image shifts due to local destretch during the scan (in the continuum outside the line) we performed a correlation analysis on small subfields of different size $(16 \times 16$ pixel, $32 \times 32$ pixel, $64 \times 64$ pixel $)$ outside the spot. For all subfield sizes the shifts in both directions are well below 1 pixel, corresponding to spatial scales of a fraction of $0.089 \mathrm{arcsec}$. The transparency (measured in the broad-band channel) varies between 0.97 and 1.01 of the total intensity in the considered subfield averaged over the whole scan. By visual inspection of the best continuum filtergram we estimate that the smallest structures have a full-width-half-maximum size of 4 pixel, corresponding to about 0.4 arcsec. For comparison, the corresponding small structures in the mean intensity map averaged over all continuum wavelength points in the scan show a full-width-half-maximum size of 5 pixel, i.e., about 0.5 arcsec, which demonstrates that the image stability and real-time seeing correction worked rather stable.

\section{Line profile analysis}

The data pre-processing results in line profiles at each spatial position. To reduce noise, we take advantage of the spatial oversampling and perform a $2 \times 2$ binning of the data cube. Physical parameters are determined at each image point and stored into maps. We derive the line depression, the equivalent width $(E W)$, the line-core intensity, and velocities along the line of sight. The line core intensity is determined by the minimum value of a parabolic fit (of 15 points centered around the minimum of the observed profile) to the line core and is given in units of the mean continuum intensity outside the spot. The line depression is calculated from the intensity difference of the local continuum and the line core, normalized to the local continuum intensity. The $E W$ results from integration of the line depression as a function of wavelength. For each line profile, 40 bisector positions are calculated at intensity levels between $2 \%$ and $80 \%$ of the line depression. The individual bisector positions result from a linear interpolation between the relevant intensities in the observed line profile and the corresponding wavelength positions. The bisectors are used to
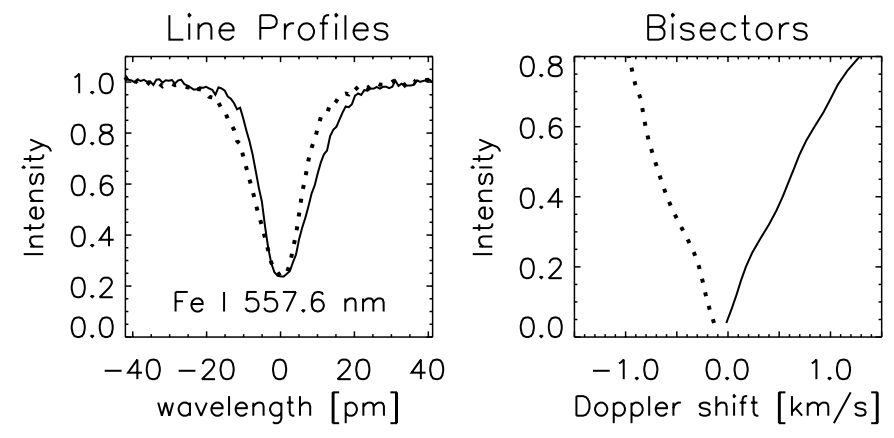

Fig. 4. Mean line profiles and their bisectors of center (dotted) and limb side (solid) penumbra. The profiles stem from the regions that are marked with black squares in Fig. 6.

determine (a) the full-width-at-half-maximum $(L W)$ of the line profile by averaging the bisector positions between $45 \%$ and $55 \%$ of the line depression, (b) the line-core Doppler shift by averaging the bisector positions between the $2 \%$ and $15 \%$ level of the line depression (we checked that line-core positions determined by a parabolic fit to the line core give the same results) and (c) the line-wing Doppler shift by averaging between $60 \%$ and $80 \%$ of the line depression. We do not consider bisector levels larger than $80 \%$ since the bisector positions in the extreme line wing are very sensitive to noise and to the residuals of the modulation pattern mentioned in Sect. 3.1. The velocity sign is defined in the following way: positive (dark) velocities point away from the observer and correspond to redshifts, while negative velocities (bright) point towards the observer and correspond to blue-shifts. In the following the terms bisectorgram and Dopplergram are used as synonyms.

Figure 4 shows two bisectors (right) obtained from spatially averaged profiles (left) taken from the limb- and center-side penumbra, respectively. The regions used for the averaging are indicated in Fig. 6 by black squares. The profiles show very nicely the characteristics of the Evershed effect: almost no relative line-core shift and a strong line asymmetry due to blueshifted and red-shifted line wings on the center- and limb-side penumbra, respectively.

At this point we want to mention that the resolution element may contain more than one atmospheric component with different filling factors. These components may carry a flow or not. Although the Fe I $557.6 \mathrm{~nm}$ is not affected by the presence of magnetic fields, it is modified by plasma flows aligned with the field lines (Bellot Rubio et al. 2003). Therefore, the unresolved fine structure of the magnetic field and the inherent flow do influence the line parameters, at least indirectly. The measured line parameters thus contain information about different atmospheric components, but it is far from trivial to separate these contributions without additional observables.

\subsection{Velocity calibration}

The velocity measurements are relative and not absolute since we do not record a wavelength reference. However, a wavelength reference must be chosen to calibrate the observed Doppler shifts. Typically, either the mean granulation or the mean umbra serves as a rest frame. We decided to take the 

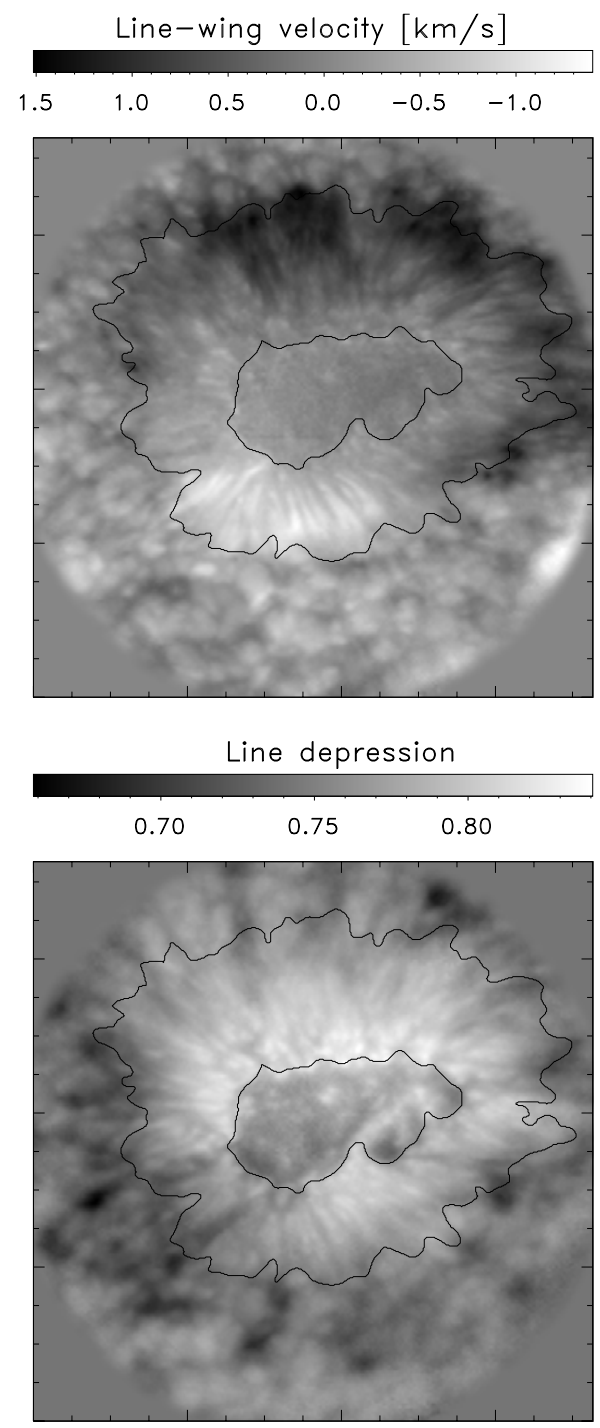
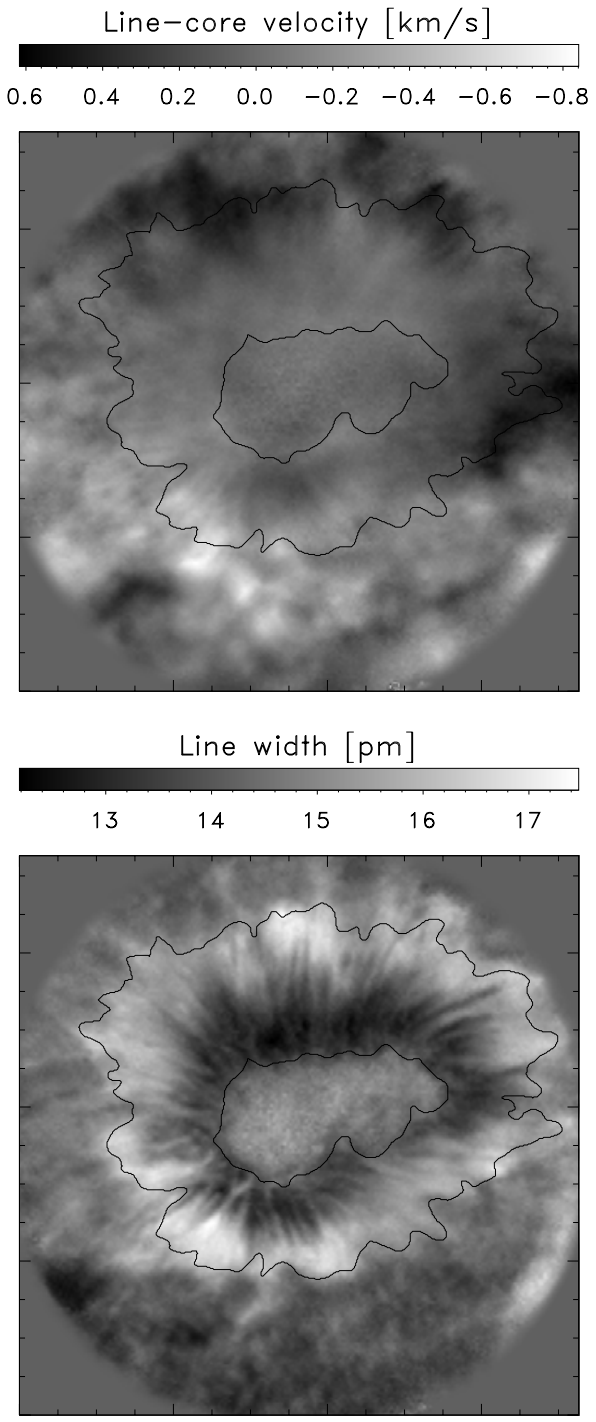
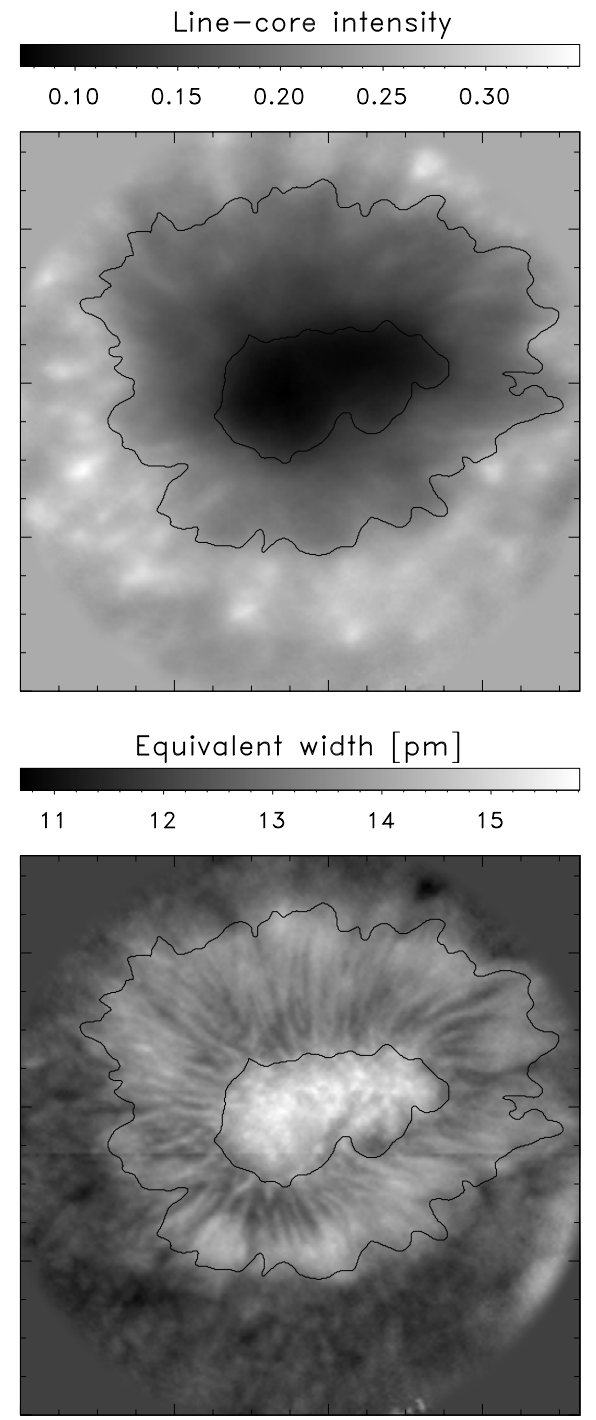

Fig. 5. Upper left to lower right: line-wing bisectorgram, line-core bisectorgram, line-core intensity, line depression, the $L W$ of the line profile and the $E W$. The line-core intensity is given in units of the mean continuum intensity outside the sunspot. The contour lines mark the inner and outer penumbral border. One minor tick mark corresponds to 5 arcsec.

mean granulation as a frame of reference and proceeded in the following way.

In a first step the two-component quiet-sun model of Borrero \& Bellot Rubio (2002) is used to determine the convective blueshift of the Fe I $557.6 \mathrm{~nm}$ line. Beside temperatures, this model contains information about the height variation of the granular and intergranular velocities in the quiet solar atmosphere. We compute two synthetic line profiles of the Fe I $557.6 \mathrm{~nm}$ line at disk center, one including the above mentioned height dependence of the granular and intergranular velocities and one with the velocities artificially set to zero. The wavelength difference between the line-core positions of the two synthetic profiles directly gives a convective blueshift for the Fe I $557.6 \mathrm{~nm}$ line of $-240 \mathrm{~m} \mathrm{~s}^{-1}$. According to Balthasar (1988) this disk-center value would be reduced by less than $10 \mathrm{~m} \mathrm{~s}^{-1}$ at the heliocentric position of our spot. In a second step, we determined the mean quiet-sun profile from a $12 \times 16 \operatorname{arcsec}^{2}$ subfield extracted from the additional scans taken slightly off the spot. The line-core position of this mean profile was set to $-240 \mathrm{~m} \mathrm{~s}^{-1}$. Using this calibration, a mean umbral profile averaged over the darkest umbral regions shows a blue-shift of $-120 \mathrm{~m} \mathrm{~s}^{-1}$ and $-40 \mathrm{~m} \mathrm{~s}^{-1}$ in the line core and the line wing, respectively. For the centerwards directed granular region indicated in Fig. 6 we find a mean blue-shift of $-335 \mathrm{~m} \mathrm{~s}^{-1}$, which is $\sim 100 \mathrm{~m} \mathrm{~s}^{-1}$ larger than the convective blue-shift used for setting the reference position. We ascribe this surplus to the influence of the moat flow.

\section{Results}

\subsection{Line parameter maps}

Figure 5 displays the following line parameter maps: line-wing velocity map (a), line-core velocity map (b), line-core intensity (c), line depression (d), line width $(L W)(\mathrm{e})$, and equivalent width $(E W)(f)$. Penumbral borders are usually defined by an intensity threshold value, which introduces some arbitrariness. We perform a triple Gaussian fit to the intensity distribution 


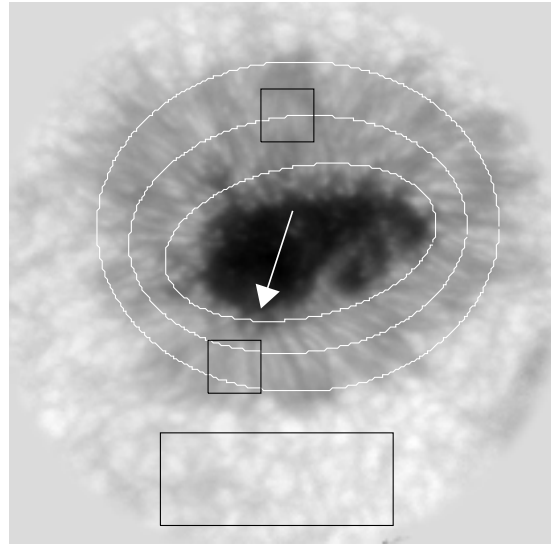

Fig. 6. Elliptical cuts with increasing semimajor axis to trace the inner, middle, and outer penumbra. The axes amount to $0.65 R, 0.80 R$, and $0.95 R$. The arrow points toward disk center. The black rectangle marks the granular region used to determine the mean quiet-sun intensity and the granular blue-shift. The two small black squares indicate the areas used for the averaged line profiles of Fig. 4.

in the continuum and define the penumbra by those intensity values that lie in the range $\left[I_{\max }-4 \sigma, I_{\max }-\sigma\right]$, where $I_{\max }$ is the peak intensity and $\sigma$ is the width of the penumbral component. In units of the observed mean quiet-sun continuum intensity, the threshold intensities correspond to 0.58 and 0.89 , respectively. These values are used to define the contour lines of Fig. 5.

The mean line-wing bisectorgram (Fig. 5a), representative for the deep layers of the penumbral atmosphere, uncovers a conspicuous filamentary-structured penumbral flow pattern and the typical properties of the Evershed flow, i.e. red-shifts (dark) on the limb-side penumbra and blue-shifts (bright) on the center-side penumbra. At the inner limb-side penumbral boundary, patches of blue-shifts (upflows) can be seen. Limbwards the maximum red-shift is observed in the outer penumbra and reaches values of up to $1.8 \mathrm{~km} \mathrm{~s}^{-1}$, while centerwards the largest blue-shifts are located in the middle penumbra and reach absolute values of up to $1.3 \mathrm{~km} \mathrm{~s}^{-1}$. On the center-side penumbra the velocity map exhibits distinct (flow-) filaments that are much less developed on the limb-side penumbra. Decisive for higher atmospheric layers of the penumbra, the mean line-core bisectorgram shows a more diffuse flow pattern dominated by the Evershed flow located in the middle part of the penumbra and beyond. The inferred velocities are in the range -0.8 to $0.7 \mathrm{~km} \mathrm{~s}^{-1}$. From the line-wing bisectorgram it is clear that the flow is well confined to the penumbra, while from the line-core bisectorgram it is obvious that there exist extensions that reach beyond the visible boundary of the penumbra. Both statements depend on the location of the penumbral border and no unambiguous conclusions can be drawn except that the flow field slightly spreads and fans out with height.

The $L W$ and the $E W$ maps show a significant radial dependence and small-scale azimuthal fluctuations that form a filamentary structure. Both quantities, in particular the $L W$, are enhanced in the outer and reduced in the inner penumbral regions. This relation has been already reported by Johannesson (1993) and Rimmele (1995a). The mean umbral value of the $L W$ is comparable to that of the quiet-sun. In contrast, the $E W$ of umbral profiles is significantly enlarged compared to those of the surrounding penumbral and granular regions even if the umbral blend in the blue wing is not accounted for. This finding is consistent with Holmes (1963) and with synthetic line calculations that we have performed by using common umbral, penumbral, and quiet-sun model atmospheres. Yet, Johannesson (1993) and Rimmele (1995a) find a different behavior for the $E W$.

The line-core intensity map shows little contrast on small scales. The umbra and the penumbra are homogeneous and unstructured in brightness with a minimum intensity inside the umbra. The latter is again consistent with Holmes (1963) and Johannesson (1993) but in contradiction to Rimmele (1995a). On average, the intensity increases from the umbra through the penumbra to reach maximum values in brightenings that are located in the immediate surrounding outside the spot. The line depression mirrors both the line-core intensity and the continuum intensity. As a result, features that belong to different heights in the solar atmosphere are visualized and combined in one single map: the filamentary structure of the penumbra is recovered and the brightenings outside the spot are visible as patches where the line is weakened. The line depression is enhanced throughout the whole penumbra and in particular the fine structure located in the inner limb-side penumbra shows a peculiar line strengthening. Although the line depression does not point at a significant azimuthal asymmetry, our visual impression is that the line is less strengthened on the center-side penumbra than on the limb-side penumbra. The mean umbral line depression is mostly unchanged compared to the spot surroundings.

\subsection{Radial dependence}

In order to work out the radial dependences, elliptical cuts are used to trace the azimuthal variations of the physical parameters. The ellipses are chosen to match concentric circumferences in the sunspot. Three sample ellipses in the inner, middle and outer penumbra are shown in Fig. 6. Radial distances correspond to the semimajor axes normalized to the axis $R$ of the ellipse fitting the outer penumbral boundary taken from the continuum. The outer penumbral boundary is defined by the contour lines indicated in Fig. 5. Along each ellipse the azimuthal average of the line parameters is computed to obtain radial profiles. The result is shown in Fig. 7 for the $E W$ (left), the $L W$ (right) and in Fig. 8 for the intensity at selected wavelength positions. The shaded areas mark the rms-fluctuations $(1 \sigma)$ of the individual values entering the azimuthal averages, and thus inform about the fine structure. It is striking that both the $E W$ and the $L W$ increase from a minimum in the inner $(r \approx 0.65 R)$ to a maximum in the outer $(r \approx 0.95 R)$ penumbra. For the $E W$ the extrema are local while they are global for the $L W$. The increase of these two quantities across the penumbra correlates with the corresponding increase of the modulus of the Doppler shifts apparent from Fig. 5a. A possible explanation for this behavior is given in Sect. 5.5. 

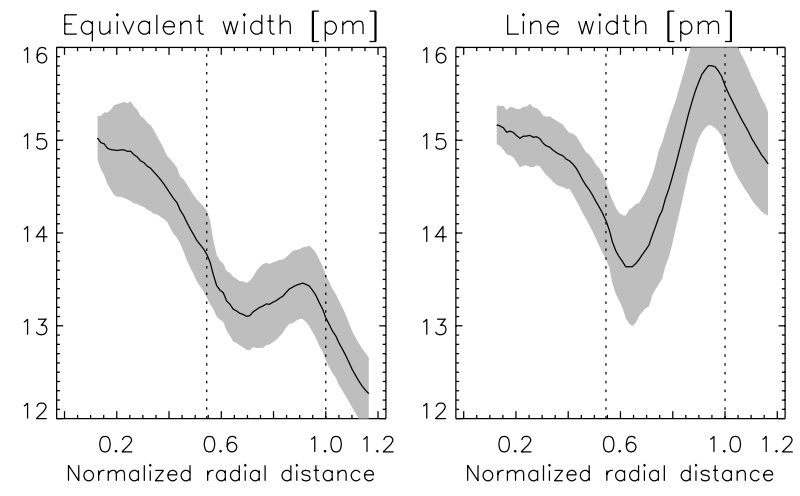

Fig. 7. Radial dependence of the azimuthally averaged $E W(l e f t)$ and $L W$ (right). The radial distance is normalized to the outer penumbral radius as seen in the continuum images. The vertical lines mark the position of the inner and outer penumbral boundaries. Shaded areas correspond to the standard deviation $(1 \sigma)$ of the azimuthally averaged values.

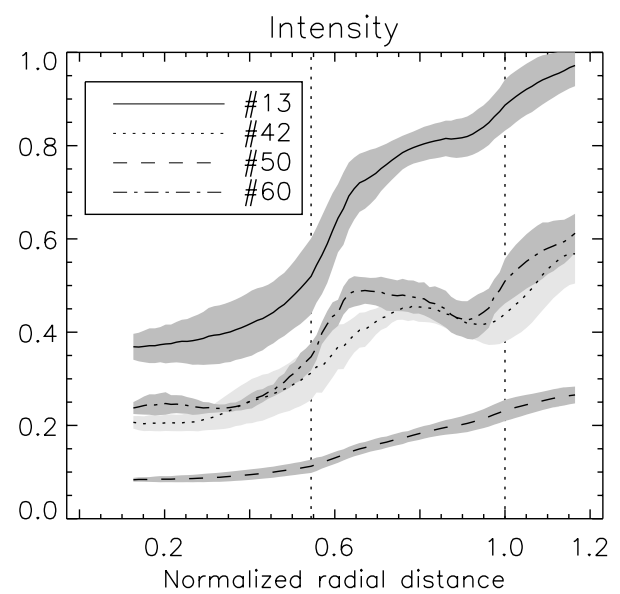

Fig. 8. Radial dependence of the azimuthally averaged intensity given in units of the mean quiet-sun intensity determined from the rectangle in Fig. 6. The numbers correspond to the filtergram numbers in Fig. 3. \#13: best continuum frame. \#42: blue wing. \#50: close to the line core. $\# 60$ : red wing. For \#42 and \#60 the averaging is done only on the center-side and limb-side penumbra, respectively. The vertical lines mark the position of the inner and outer penumbral boundaries.

In Fig. 3, the line-wing intensity (filtergrams \#42 and \#60) exhibits a similar behavior even though not that pronounced: the inner penumbral brightness is enhanced compared to the outer penumbra. For better visibility of this effect, the intensity average in the blue wing (\#42) and in the red wing (\#60) is performed only on the center-side and limb-side penumbra, respectively. In contrast, the continuum intensity (\#13) and the line-core intensity (\#50) show a monotonic increase in intensity from the umbra to the outer penumbra.

This "bright ring" in the inner penumbra is also directly seen in the corresponding filtergrams displayed in Fig. 3. A similar ring has been detected also from narrowband filtergrams near the core of the Fe I $557.6 \mathrm{~nm}$ line by Balasubramaniam (2002) (see Fig. 8 therein) although the phenomenon is not mentioned by the author. The most prominent bright ring is apparent from spectropolarimetric observations at $1564.8 \mathrm{~nm}$ taken with the TIP instrument and discussed in
Bellot Rubio (2003). Hitherto, the enhanced line-wing brightness in the inner penumbra is not understood. It is tempting to explain this phenomenon as an indication of temperature enhancements in the middle photospheric layers (cf. Bellot Rubio 2003), but it may also be understood in terms of Doppler shifts. This will be demonstrated in a forthcoming paper.

\subsection{Azimuthal variation}

Figure 9 shows the azimuthal variation of the line parameters along three ellipses (see Fig. 6) placed at different radial distances: in the inner (left panels, $r=0.65 R$ ), the middle (central panels, $r=0.80 R$ ), and the outer (right panels, $r=0.95 R$ ) penumbra. From top to bottom, we plot the azimuthal variation of the line-wing velocity, $v_{\mathrm{LOS}}$, the normalized continuum intensity, the $E W$, the $L W$, and the line depression. The azimuthal angle, $\phi$, is measured counter-clockwise with $\phi=90^{\circ}$ and $\phi=270^{\circ}$ pointing to the limb and disk center, respectively. The coarse azimuthal variation of the velocity gradually increases towards the outer penumbra and shows demonstratively the increase of the Evershed flow with radial distance. This behavior is very well characterized by a sinusoidal dependence of the form

$f(\phi)=A \cdot \sin (\phi)+m$,

where $m$ denotes the azimuthal average (dotted horizontal line in Fig. 9). The result of the least-squares fit is indicated by the dashed lines in Fig. 9. Small-scale fluctuations of the velocity ascribed to the penumbral fine structure are superposed to the sinusoidal azimuthal variation. In contrast to the velocity, the other line parameters show no distinct dependence on azimuth, although in the outer penumbra the $E W$ and the $L W$ tend to be slightly enhanced in the regions of maximum absolute velocity (as indicated by the curved lines of the corresponding plots). This conjecture will be addressed again in Sect. 5.5.

\subsection{Flow geometry}

Under the assumption that the flow field within the penumbra is a function of radius and azimuth only, the azimuthally averaged flow geometry can be deduced from the azimuthal dependence of the LOS velocity (see e.g., Schröter 1965; Title et al. 1993; Schlichenmaier \& Schmidt 2000). In particular, this assumption implies that the velocity fluctuations ascribed to the penumbral fine structure are small compared to the overall azimuthal dependence caused by the projection of the flow vector onto the LOS. These assumptions seem to be satisfied inspecting the sample slices of Fig. 9. Another assumption is that the flow fills the whole resolution element. Presuming that the flow field is axially symmetric, the LOS component of the velocity vector is given by

$v_{\mathrm{LOS}}(r, \phi)=v_{\mathrm{h}}(r) \sin \theta \sin \phi+v_{\mathrm{v}}(r) \cos \theta$,

where $\theta$ is the heliocentric angle of the sunspot, and $v_{\mathrm{h}}$ and $v_{\mathrm{v}}$ denote the horizontal and vertical velocity components, respectively. According to Eq. (2) the vertical component can be deduced from the azimuthal average of the LOS velocity 

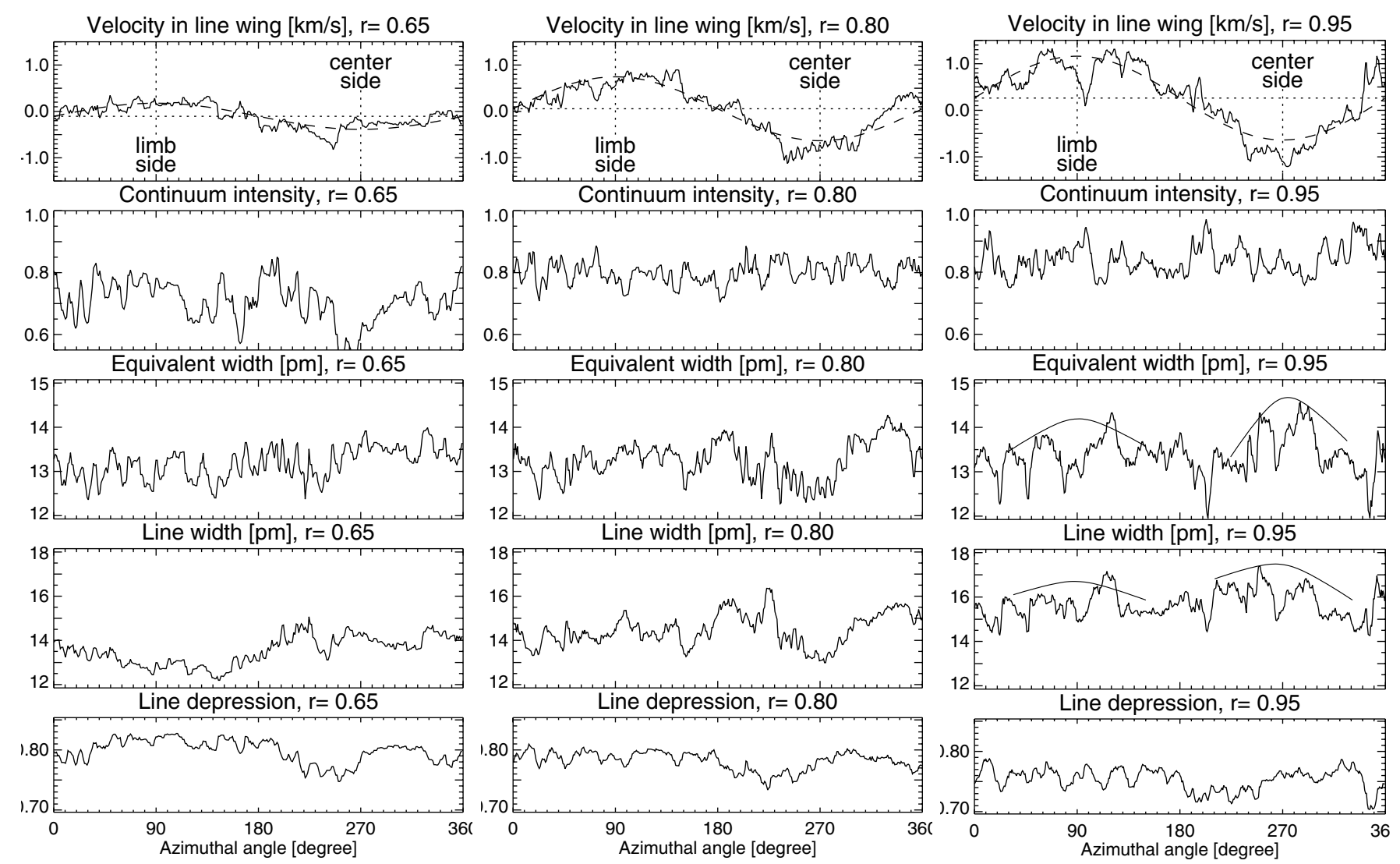

Fig. 9. From top to bottom: azimuthal variation of the line-wing velocity, the continuum intensity, the $E W$, the $L W$ and the line depression extracted from the ellipses shown in Fig. 6 . The azimuthal angle, $\phi$, is measured counter-clockwise, with $\phi=270^{\circ}$ pointing towards disk center.

$\left\langle v_{\mathrm{LOS}}\right\rangle_{\phi}=m=v_{\mathrm{v}} \cos \theta$, while the horizontal component is inferred from the amplitude, $A=v_{\mathrm{h}} \sin \theta$, of the sinusoidal leastsquares fit to the observed $v_{\text {LOS }}$ (see Eq. (1)). Knowledge of $v_{\mathrm{h}}$ and $v_{\mathrm{v}}$ allows us to calculate the mean flow angle, $\gamma$, and the absolute flow velocity, $v_{0}$, via the relations $\gamma=\tan ^{-1}\left(v_{\mathrm{h}} / v_{\mathrm{v}}\right)$ and $v_{0}=\sqrt{v_{\mathrm{h}}^{2}+v_{\mathrm{v}}^{2}}$, respectively.

All components that determine the velocity vector are shown in Fig. 10 as a function of radial distance from the center of the spot: the inclination angle (left), the absolute velocity (right, solid), the amplitude of the sinusoidal fit (right, dashed) and the azimuthal mean of the LOS velocity (right, dotted). As input for the LOS velocity we use the mean line-wing bisectorgram (cf. Sect. 5.1).

A negative value for the vertical component implies an upflow $\left(\gamma<90^{\circ}\right)$ and a positive value implies a downflow $\left(\gamma>90^{\circ}\right)$. In the inner penumbra we find upflow components of up to $\sim 0.1 \mathrm{~km} \mathrm{~s}^{-1}$. At a radial distance of $r \approx 0.75 R$, the vertical component vanishes and becomes negative in the outer penumbra reaching a maximum at $r \approx 0.95 R$ of $-0.25 \mathrm{~km} \mathrm{~s}^{-1}$. The amplitude, $A$, being positive by definition has a maximum of $\sim 1.0 \mathrm{~km} \mathrm{~s}^{-1}$ at $r \approx 0.90 R$. Consequently, the flow angle, $\gamma$, increases monotonically from $\sim 60^{\circ}$ in the inner penumbra to slightly more than $95^{\circ}$ at $r \approx 0.95 R$. The absolute velocity has its maximum value of $v_{0}=2.5 \mathrm{~km} \mathrm{~s}^{-1}$ at $r \approx 0.90 R$. The determination of the velocity vector critically depends on the velocity calibration and one should recall that in the present investigation the mean granulation serves as a frame of rest.
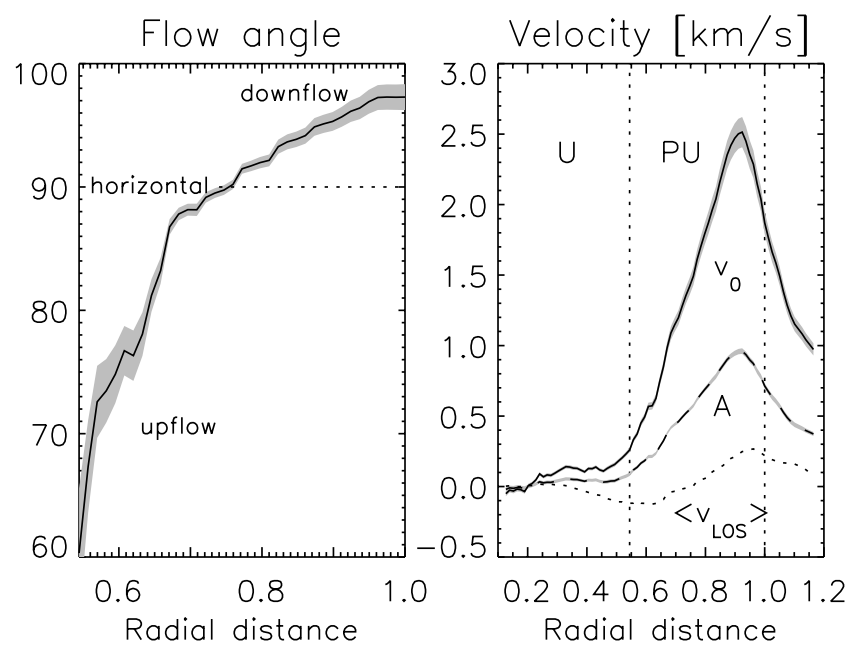

Fig. 10. Radial dependence of the mean flow vector. Left: the mean flow angle, $\gamma$, in the penumbra. Right: the absolute velocity, $v_{0}$, (solid line), amplitude, $A$, of the sinusoidal fit (dashed) and the azimuthal average, $\left\langle v_{\mathrm{LOS}}\right\rangle_{\phi}$, of the LOS velocity (dotted). The vertical dotted lines mark the inner and the outer penumbral boundary. The shaded areas give the $1 \sigma$ uncertainty estimate for the fits and the corresponding uncertainties computed by error propagation.

Hence, it is remarkable that our results agree so well with the findings of Schlichenmaier \& Schmidt (2000) even though these authors used another spectral line. Our results are also in excellent agreement with the two-component inversions of 
Doppler shift in line wing
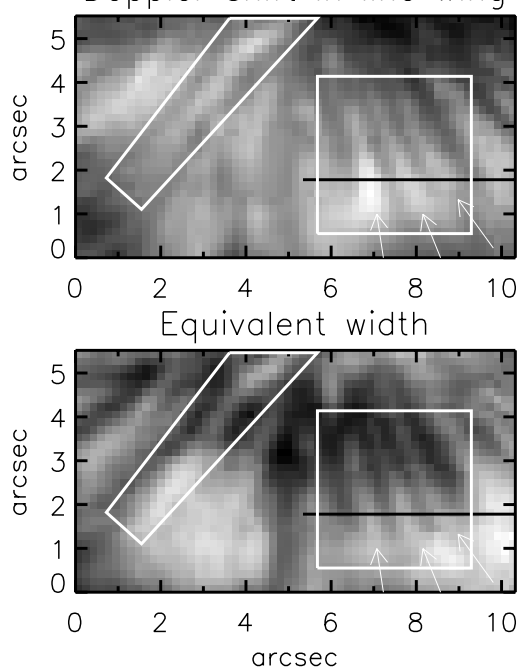
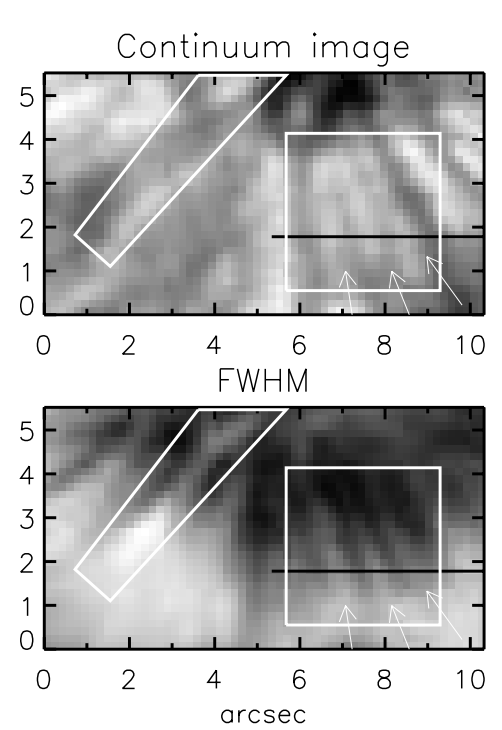
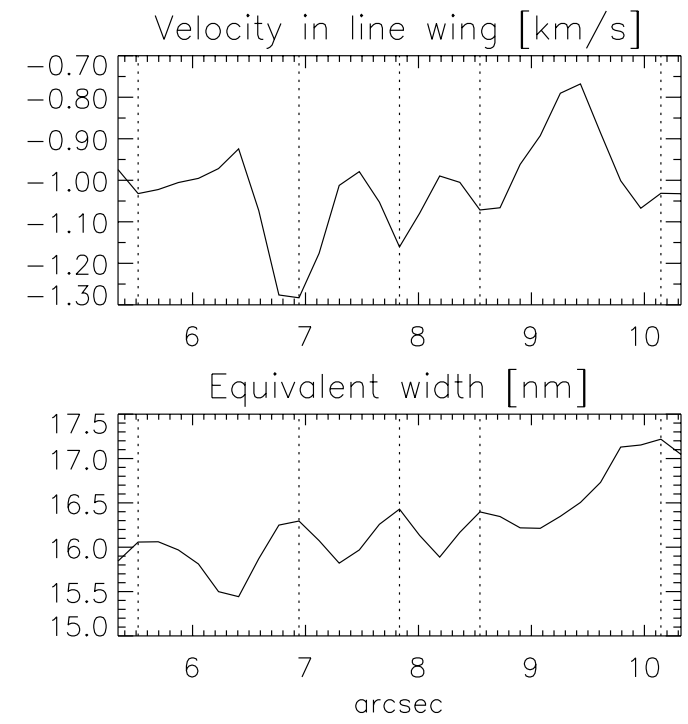

Fig. 11. Left: close-up look of the center-side penumbra. The four maps display from the upper left to the lower right: the line-wing velocity, the continuum intensity, the $E W$, and the $L W$, respectively. The contrast of the continuum image is slightly enhanced. Right: line-wing velocity (top) and $E W$ (bottom) along the cut indicated by the black line in the subfield regions.

Bellot Rubio et al. (2003), who found that the velocity vector and the magnetic field vector of the atmospheric component carrying the Evershed flow return to the solar surface in the middle penumbra and beyond. Therefore, we consider the presence of a downflow component in the outer penumbra as a solid result.

\subsection{Individual features of interest}

\subsubsection{Flow filaments on disk center side}

The demonstrable filamentary nature of the penumbral flow field (cf. Fig. 5) warrants a closer look. Therefore, Fig. 11 highlights a subfield region located on the center-side penumbra. Displayed are the line-wing velocity, the continuum intensity (contrast enhanced), the $E W$ and the $L W$. The orientation is the same as in Fig. 5. The white lines outline two areas that show elongated flow filaments: an individual flow filament (tetragon, upper left) and three adjacent flow filaments (square, lower right).

The single flow filament (tetragon) starts in the inner penumbra with an enhanced brightness (penumbral grain). In the outer course of the flow filament, the corresponding intensity is significantly reduced w.r.t. the local average. A possible explanation for this behavior can be given within the framework of the moving-tube model suggested by Schlichenmaier et al. (1998). In this scenario, penumbral grains are interpreted as the upflow footpoints of ascending flux tubes located in the inner penumbra. Confined to the magnetic flux tube the hot plasma flow is directed horizontally and radially outwards. When entering the photosphere the optically thick plasma cools by radiative heat exchange until the flux tube becomes optically thin (Schlichenmaier et al. 1999) and thus undistinguishable from the surrounding background plasma.

The three adjacent filaments (square) behave similarly. The flow filaments, but also the corresponding signal in $E W$ and
$L W$, widen with radial distance. In the outer penumbra, the flow filaments reach the maximum Doppler shift and tend to be co-spatial with dark intensity structures and local increases in the $E W$ and the $L W$. The co-spatiality and good correlation is demonstrated by the two diagrams in the right panel of Fig. 11 for the variation of the velocity (upper panel) and the $E W$ (lower panel) along the black line crossing the three adjacent structures.

Hence, we find a correlation between the modulus of the line-of-sight velocity, the $E W$, and the $L W$ on small spatial scales along azimuthal cuts (that cross the filamentary structure) and, additionally, an increase of the azimuthal averages of the corresponding quantities with radial distance (cf. Fig. 7 and Fig. 10).

In principle, enhancements of $E W$ and $L W$ for a line as strong as Fe I $557.6 \mathrm{~nm}$ may be due to micro-turbulence, but since we find a good correlation with enhancements of flow velocity, we conjecture that the observed enhancements of $E W$ and $L W$ can at least be partly ascribed to the presence of a Doppler-shifted line satellite. We have calculated synthetic line profiles of Fe I $557.6 \mathrm{~nm}$ to demonstrate that a deep-lying flow channel (extending from -1.5 to -0.5 in $\log \tau$, with a line-ofsight velocity of $1 \mathrm{~km} \mathrm{~s}^{-1}$ ) embedded in an atmosphere at rest broadens the line by $6 \%$ (due to the line satellite), while the line depth remains unchanged, and the $E W$ increases by $4 \%$, i.e., the $E W$ and $L W$ are significantly enhanced compared to the profile where no flow channel is present. These considerations indicate that micro-turbulence may not be the only source of enhanced $E W$ and $L W$, but that directed flows also play an essential role. In order to distinguish between the two effects, we note that directed flows should lead to a projection effect, i.e. an azimuthal variation, for spots at large heliocentric angles, while micro-turbulence is isotropic. The heliocentric angle of the spot that we investigate here is too small to show a clear azimuthal variation in $E W$ and $L W$, although as mentioned in Sect. 5.3, Fig. 9 (right panel) exhibits the trend of an 

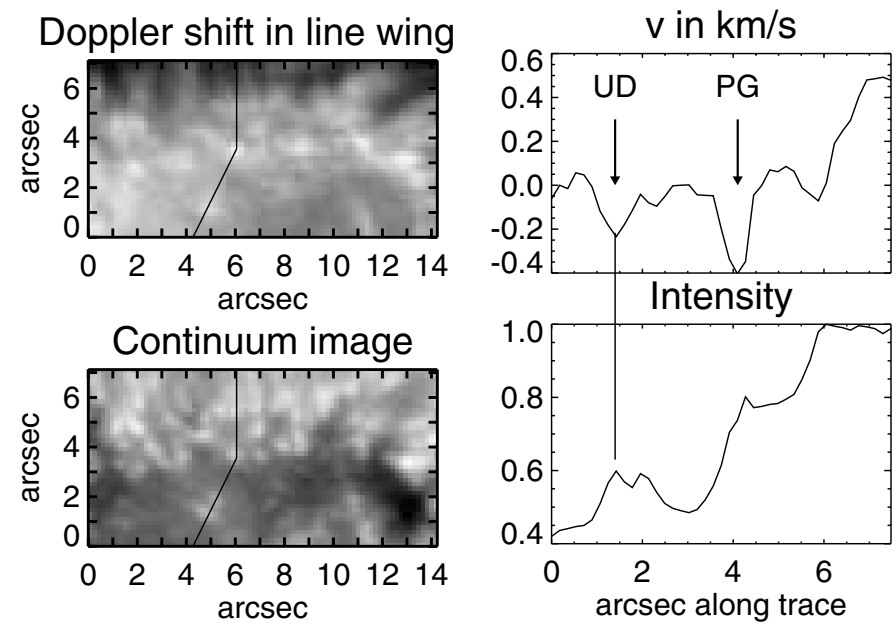

Fig. 12. Left: close-up look of the umbra and the inner limb-side penumbra. The two images show the line-wing velocity and the continuum intensity (unsharp masked). Right: line-wing velocity (top) and intensity (bottom) along the cut indicated by the line in the subfield regions.

enhancement in $E W$ and $L W$ for the outer limb and center-side penumbra.

\subsubsection{Umbral dots and penumbral grains}

While the filamentary structure of the line-wing bisectorgram (cf. Fig. 5a) is less pronounced on the limb side, penumbral grains are more distinct on this side and are hardly seen on the center-side penumbra. Figure 12 presents a subfield showing penumbral grains located at the inner limb-side penumbra. Brightenings in intensity (i.e., penumbral grains) tend to be correlated with blue-shifts. We follow the intensity and velocity signal along a cut through the umbra and the inner penumbra marked in Fig. 12. The cut intersects two umbral dots (UD) and one penumbral grain (PG).

The penumbral grain (at $\sim 4 \operatorname{arcsec}$ ) is almost hidden in the intensity plot due to the strong intensity gradient towards the penumbra. In the intensity map (unsharp masked) the penumbral grain is clearly visible. The blue-shift associated with the penumbral grain amounts to $-400 \mathrm{~m} \mathrm{~s}^{-1}$. The direct detection of vertical flow components is restricted to disk-center observations and the interpretation of the above mentioned blueshift in terms of an upflow is hampered due to the presence of proper motions of penumbral grains. Sobotka et al. (1999) find a single-peaked distribution of time-averaged proper motion speeds for penumbral grains: $79 \%$ of all observed inward moving penumbral grains have proper motion speeds in the range $100-700 \mathrm{~m} \mathrm{~s}^{-1}$ with a typical speed of roughly $\sim 400 \mathrm{~m} \mathrm{~s}^{-1}$. Higher speeds $\left(>700 \mathrm{~m} \mathrm{~s}^{-1}\right)$ refer to penumbral grains that are located at larger radial distances $(r>0.6 R)$. The projection of a proper motion speed of $\sim 400 \mathrm{~m} \mathrm{~s}^{-1}$ onto our line-of-sight leads to a blue-shifted component of $\sim-150 \mathrm{~m} \mathrm{~s}^{-1}$. Thus, the proper motion cannot account alone for the observed blue-shift and we therefore interpret the blue-shift associated with the penumbral grain as partially produced by an upflow. Hence all the brightenings (blue-shifts) that are seen in the velocity map of the inner limb-side penumbra represent partially upflows, that may serve as a source to supply the horizontal flow component of the Evershed flow. This indicates that penumbral grains are formed by hot upflowing plasma that radiatively cools after entering the photosphere where radiative relaxation is very efficient.

In the intensity map, two umbral dots show up next to each other. At the location of these two umbral dots the velocity map reveals one blue-shifted patch. While the two umbral dots are well distinguishable in intensity, only one umbral dot is associated with a blue-shift of $-200 \mathrm{~m} \mathrm{~s}^{-1}$ in the velocity map. Both umbral dots show up as enhancements in $E W$ and line depth (not displayed here), but we note that both umbral dots and the penumbral grain do not show a corresponding signal in the line width $(L W)$. Since the spatial resolution of the velocity map is better than 0.5 arcsec, while the separation of the two dots in the intensity map is more than 0.5 arcsec, we conclude that one of the two umbral dots shows a blue-shift while the other one does not show a significant velocity signal. In contrast to penumbral grains, the distribution of time-averaged proper motion speeds for umbral dots does not show an isolated peak, but gradually decreases with increasing speed: speeds are in the range $100 \mathrm{~m} \mathrm{~s}^{-1}-1000 \mathrm{~m} \mathrm{~s}^{-1}$ without any dependence on radial distance (Sobotka et al. 1997). This leads to line-of-sight velocities that do not allow to draw any conclusions about the observed blue-shift in umbral dots in terms of upflows and/or proper motions without further information.

\section{Summary and discussion}

We report on the analysis of a high-quality data set acquired at the German VTT on Tenerife with the 2D-spectrometer TESOS, equipped with three Fabry-Perot-Interferometers (etalons) and backside-illuminated Pixel Vision CCDs that allow exposure times as small as $10 \mathrm{~ms}$ for individual filtergrams. Scanning 100 wavelength points is accomplished within $37 \mathrm{~s}$. Further, this was the first scientific observing campaign that employed the Kiepenheuer Adaptive Optics System (KAOS).

As the scientific target, we have selected the fine-structure and spectroscopic signatures of a sunspot penumbra. We have observed a spot at a heliocentric angle of $22.6^{\circ}$ using the magnetically insensitive absorption line of neutral iron at $557.6 \mathrm{~nm}$, achieving a spatial resolution of better than half an arcsec and a spectral resolution of $\lambda / \delta \lambda=250000$. We have analyzed line parameter maps, their radial and azimuthal dependence within the penumbra, inferred the flow geometry, and discussed individual features of interest. Our main results can be summarized as follows:

1. The line-wing bisectorgram shows a remarkable filamentary penumbral flow field. On the center-side penumbra, individual flow filaments are discernable, but not on the limb-side penumbra. In contrast thereto, the line-core bisectorgram has lower contrast and does not carry signatures of the penumbral fine-structure.

2. The Evershed flow in the deep atmospheric layers (linewing bisectorgram) is well confined to the continuum 
penumbra. In higher penumbral layers (line-core bisectorgram) the flow field shows extensions beyond the visible boundary of the penumbra that are more pronounced on the limb-side penumbra.

3. We infer the flow geometry from the azimuthal dependence of the LOS component of the velocity and find that the absolute flow velocity increases gradually from the inner towards the outer penumbral boundary and then drops off. The maximum value of the flow velocity $\left(v_{0}=2.5 \mathrm{~km} \mathrm{~s}^{-1}\right)$ is reached at $r=0.9 R$ in the outer penumbra. Reflecting the vertical component of the velocity, the azimuthally averaged LOS velocity indicates the existence of an upflow component in the inner penumbra and a downflow component in the middle and outer penumbra. The retrieved flow angle (measured with respect to the solar surface normal) increases from $60^{\circ}$ at the inner to $97^{\circ}$ at the outer penumbral boundary, crossing $90^{\circ}$ at $r=0.75 R$. This confirms the findings of Schlichenmaier \& Schmidt (2000) and more recent results presented by Bellot Rubio et al. (2003). Further observational evidence for the existence of downflows at the outer edge of the penumbra and beyond is given by Rimmele (1995b), Stanchfield et al. (1997), Westendorp Plaza et al. (1997), and Schmidt \& Schlichenmaier (2000).

4. Penumbral grains are predominantly seen on the inner limb-side penumbra and are associated with blue-shifts, indicating upflows (also reported by Hirzberger \& Kneer 2001). In one particular case, we measure a blue-shift of $v=-400 \mathrm{~m} \mathrm{~s}^{-1}$. The presence of upflows in bright structures is consistent with Johannesson (1993), Rimmele (1995a), Rimmele (1995b), Stanchfield et al. (1997), and Schmidt \& Schlichenmaier (2000). We interpret the observed upflows in penumbral grains as upstream footpoints of ascending magnetic flux tubes as proposed in the moving-tube model of Schlichenmaier et al. (1998).

5. Azimuthal averages of $E W, L W$ and the absolute flow velocity show an increase with radial distance from the inner towards the outer penumbra. In addition, small-scale variations of the $E W$ and the $L W$ particularly on the center-side penumbra are co-spatial with an increased modulus of the LOS velocity. We take this for evidence that the observed behavior of $E W$ and $L W$ are mainly produced by Doppler broadening due to directed flows, rather than by micro-turbulence.

6. Filtergrams taken in the line wing (intermediate intensity levels) show a well known (cf. Bellot Rubio 2003), but not yet understood, phenomenon: the inner penumbra tends to be brighter than the outer penumbra. This "bright ring" might be interpreted as an increased temperature of the middle atmospheric layers of the inner penumbra, but might as well be produced by the flow field.

7. In one umbral dot we measure a blue-shift relative to the surrounding umbra of $v=-200 \mathrm{~m} \mathrm{~s}^{-1}$. Interestingly, the intensity map shows a second umbral dot next to it, which is not associated with a velocity signal. We conclude that one umbral dot is associated with a blue-shift, while the other one is not.
Some of our observational findings (e.g. "bright ring") will be addressed in forthcoming publications based on the same data set. This will include a thorough bisector analysis by means of a forward modeling in order to reproduce and interpret the observed bisectors for different locations in the penumbra. The thermal and kinematic structure of the penumbra will be studied by an inversion of the observed line profiles.

Other findings need to be investigated with additional information. To verify the conjecture that the $E W$ and the $L W$ are mainly produced by Doppler broadening due to directed flows, one should study the center-to-limb variation of a sunspot. Further, the question whether the velocity signal of umbral dots is time dependent, or whether classes of umbral dots exist that show a blue-shift while other classes are not associated with a velocity signal can be studied only on the basis of a time sequence.

Acknowledgements. The authors are very grateful to T. Kentischer for providing and maintaining the state-of-the-art instrument TESOS. We are indepted to H. Schleicher for helping to develop and for supporting the software package TESOSLIB, which we used to reduce our data. Part of this work has been supported by the Deutsche Forschungsgemeinschaft under grants PE 782/4 (AT) and SCHL 514/2-1 (LB). The Vacuum Tower Telescope at the Observatorio del Teide on Tenerife is operated by the Kiepenheuer Institut für Sonnenphysik in Freiburg (Germany).

\section{References}

Balasubramaniam, K. S. 2002, ApJ, 575, 553

Balthasar, H. 1988, A\&AS, 72, 473

Balthasar, H., Schmidt, W., \& Wiehr, E. 1997, Sol. Phys., 171, 331

Bellot Rubio, L. 2003, Solar Polarization, ed. J. Trujillo Bueno \& J. Sanchez Almeida, ASP Conf. Ser., in press

Bellot Rubio, L. R., Collados, M., Ruiz Cobo, B., \& Rodríques Hidalgo, I. 2002, Il Nuovo Cimento C, in press

Bellot Rubio, L. R., Balthasar, H., Collados, M., \& Schlichenmaier, R. 2003, A\&A, 403, L47

Berkefeld, T., Soltau, D., Schelenz, T., \& Wöger, F. 2003, A\&A, submitted

Boerner, P., \& Kneer, F. 1992, A\&A, 259, 307

Borrero, J. M., \& Bellot Rubio, L. R. 2002, A\&A, 385, 1056

Degenhardt, D., \& Wiehr, E. 1991, A\&A, 252, 821

del Toro Iniesta, J. C., Tarbell, T. D., \& Ruiz Cobo, B. 1994, ApJ, 436, 400

del Toro Iniesta, J. C., Bellot Rubio, L. R., \& Collados, M. 2001, ApJ, 549, L139

Evershed, J. 1909, MNRAS, 69, 454

Hirzberger, J., \& Kneer, F. 2001, A\&A, 378, 1078

Holmes, J. 1963, MNRAS, 126, 155

Johannesson, A. 1993, A\&A, 273, 633

Kentischer, T. J., Schmidt, W., Sigwarth, M., \& Uexkuell, M. V. 1998, A\&A, 340, 569

Martinez Pillet, V. 1997, 1st Advances in Solar Physics Euroconference. Advances in Physics of Sunspots, ed. B. Schmieder, J. C. del Toro Iniesta, \& M. Vazquez, ASP Conf. Ser., 118, 212

Mathew, S. K., Lagg, A., Solanki, S. K., et al. 2003, A\&A, 410, 695

Rimmele, T. R. 1995a, A\&A, 298, 260

Rimmele, T. R. 1995b, ApJ, 445, 511

Rouppe van der Voort, L. H. M. 2002, A\&A, 389, 1020 
Ruiz Cobo, B., \& del Toro Iniesta, J. C. 1992, ApJ, 398, 375

Scharmer, G. B., Gudiksen, B. V., Kiselman, D., Löfdahl, M. G., \& Rouppe van der Voort, L. H. M. 2002, Nature, 420, 151

Schlichenmaier, R. 2003, Current theoretical models and future high resolution solar observations, ed. A. Pevtsov \& H. Uitenbroek, ASP Conf. Ser., 286, 211

Schlichenmaier, R., \& Schmidt, W. 1999, A\&A, 349, L37

Schlichenmaier, R., \& Schmidt, W. 2000, A\&A, 358, 1122

Schlichenmaier, R., Jahn, K., \& Schmidt, H. U. 1998, A\&A, 337, 897

Schlichenmaier, R., Bruls, J. H. M. J., \& Schüssler, M. 1999, A\&A, 349,961

Schlichenmaier, R., Bellot Rubio, L. R., \& Tritschler, A. 2004, A\&A, 415,731

Schmidt, W., \& Schlichenmaier, R. 2000, A\&A, 364, 829

Schmidt, W., Hofmann, A., Balthasar, H., Tarbell, T. D., \& Frank, Z. A. 1992, A\&A, 264, L27

Schröter, E. H. 1965, Z. Astrophys., 62, 228

Sobotka, M., Brandt, P. N., \& Simon, G. W. 1997, A\&A, 328, 689

Sobotka, M., Brandt, P. N., \& Simon, G. W. 1999, A\&A, 348, 621

Solanki, S. K. 2003, A\&AR, 11, 153

Solanki, S. K., Montavon, C. A. P., \& Livingston, W. 1994, A\&A, 283, 221
Soltau, D., Berkefeld, T., von der Lühe, O., Wöger, F., \& Schelenz, T. 2002, AN, 323, 236

St. John, C. E. 1913, ApJ, 37, 322

Stanchfield, D. C. H., Thomas, J. H., \& Lites, B. W. 1997, ApJ, 477, 485

Stellmacher, G., \& Wiehr, E. 1980, A\&A, 82, 157

Title, A. M., Frank, Z. A., Shine, R. A., et al. 1993, ApJ, 403, 780

Tritschler, A., Schmidt, W., Langhans, K., \& Kentischer, T. 2002, Sol. Phys., 211, 17

von der Lühe, O. \& Kentischer, T. 2000, A\&AS, 146, 499

von der Lühe, O., Soltau, D., Berkefeld, T., \& Schelenz, T. 2003, in Innovative Telescopes and Instrumentation for Solar Astrophysics, ed. S. L. Keil, \& S. V. Avakyan, Proc. SPIE, 4853, 187

Westendorp Plaza, C., del Toro Iniesta, J. C., Ruiz Cobo, B., et al. 1997, Nature, 389, 47

Westendorp Plaza, C., del Toro Iniesta, J. C., Ruiz Cobo, B., et al. 2001, ApJ, 547, 1130

Wiehr, E., \& Degenhardt, D. 1992, A\&A, 259, 313

Wiehr, E., Knoelker, M., Grosser, H., \& Stellmacher, G. 1986, A\&A, 155,402 